\section{KIM-1: Friend or Foe?}

Kidney injury molecule-1 (KIM-1), a transmembrane phosphatidylserine receptor, is transiently upregulated in proximal tubule cells in acute kidney injury and expressed more consistently in chronic kidney disease and renal fibrosis. Because KIM-1 is not expressed in the normal kidney and is released in urine by injured epithelial cells, KIM-1 has been viewed as a promising biomarker of proximal tubule toxicity (1).

New research in mouse models by Humphreys, Bonventre, and colleagues shows that, more than a simple marker, KIM-1 plays a role in renal fibrosis and progression of chronic kidney disease (2). Those authors first evidenced the kinetics of KIM-1 induction during renal fibrotic disease using the model of unilateral ureteral obstruction in mice. Two days after ureteral ligation, KIM-1 was strongly induced in the apical pole of proximal tubule cells, with lower but persistent expression at 7 and 14 days. The authors next created a conditional transgenic mouse that permitted selective activation of KIM-1 in the absence of injury stimulus. By 4 weeks of age, mice expressing KIM-1 developed progressive interstitial lesions, with inflammation, fibrosis, and focal tubular damage leading to chronic kidney disease and extrarenal manifestations including anemia, cardiac hypertrophy, hypertension, and early death. Stable transfection of proximal tubule cells with KIM-1 triggered the release of various cytokines, including transforming growth factor $\beta$ and monocyte chemoattractant protein 1 (MCP-1), which were also upregulated in the transgenic KIM-1 kidneys. By contrast, mice expressing a truncated form of KIM-1 were protected from renal fibrosis induced by unilateral ureteral obstruction, and they showed a significant reduction in levels of MCP-1.

Taken together, these data indicate that chronic KIM-1 expression in kidney tubules upregulates a set of proinflammatory cytokines (including MCP-1) that might in turn drive leukocyte infiltration and kidney fibrosis. This hypothesized sequence contrasts with the early induction of KIM-1 after acute kidney injury, which is thought to facilitate tubular repair through phagocytosis of cells and debris and which is considered to be a marker of tubular injury.

To date, no link has been established between KIM-1 (chiefly expressed by tubular epithelial cells) and injury or fibrosis of the peritoneal membrane $(3,4)$. Fundamentally, Humphreys et al. showed that the sentinels of cell damage-which can be viewed primarily as biomarkersmight also play a causal role in chronic organ dysfunction, thus becoming potential therapeutic targets. Their studies also provide a paradigm for dissecting the mechanisms of tissue fibrosis, with investigation in rodents of the kinetics of expression upon injury; for generating and characterizing conditional and mutant mouse models; and for designing studies based on stably transfected cells.

\section{Olivier Devuyst} Deputy Editor

\section{REFERENCES}

1. Lim AI, Tang SC, Lai KN, Leung JC. Kidney injury molecule-1: more than just an injury marker of tubular epithelial cells? J Cell Physiol 2013; 228:917-24.

2. Humphreys BD, Xu F, Sabbisetti V, Grgic I, Naini SM, Wang $\mathrm{N}$, et al. Chronic epithelial kidney injury molecule-1 expression causes murine kidney fibrosis. J Clin Invest 2013; 123:4023-35.

3. Krediet RT, Struijk DG. Peritoneal changes in patients on longterm peritoneal dialysis. Nat Rev Nephrol 2013; 9:419-29.

4. Korte MR, Sampimon DE, Betjes MG, Krediet RT. Encapsulating peritoneal sclerosis: the state of affairs. Nat Rev Nephrol 2011; 7:528-38.

doi:10.3747/pdi.2014.00013 